\title{
Instabilités hydrauliques dans les centrales
}

\section{Hydraulic instability effects in power stations}

\section{Jean-Noël Plichon}

Contrôleur Général à la Direction de l'Équipement d'E.D.F.

\author{
André Lescure
}

Chargé de Mission à la Direction de l'Équipement d'E.D.F.

\section{RESUME DE LA COMMUNICATION}

La communication relate les instabilités hydrauliques les plus typiques observées dans les installations d'E.D.F. au cours des 20 dernières années et les modifications qui ont permis de les supprimer ou de les rendre acceptables.

Elle est présentée de la façon suivante :

Instabilités provoquées par un système de régulation :

- Pompage d'un groupe à la suite de la rupture de l'asservissement aiguille-déflecteur /Usine de Sassenage)

- Intumescences dans un canal nécéssitant dans certains cas la modification des consignes du régulateur ou l'inhibition de ses ordres (Usine de Riouperoux)

- Balancement d'une retenue par suite de la réflexion par la rive opposée des oscillations provoquées par la manouvre des clapets de réglage du niveau (Barrage des Fades)

\section{Instabilités provoquées par des tourbillons :}

- Vibrations d'un tronçon de conduite forcée compris entre deux joints de dilatation (Usine de SaintChamas)

- Vibrations d'aubes avant-directrices (Usines de Manosque et Sainte-Tulle II)

Instabilités provoquées par des vannes sur conduites :

Oscillations de pression dans des conduites provoquées par:

- Les vannes d'isolement d'un collecteur /Usine de Pragnères)

- Les vannes de garde des turbines (Usine de Goule Blanche)
Instabilités provoquées par des turbines

- Vibrations d'une conduite forcée à toutes les charges de la turbine (Usine de Corscia)

- Vibrations d'une conduite forcée au cours des démarrages en pompe et en turbine (Usine de La Coche)

\section{EXTRAITS DE LA COMMUNICATION $\left(^{(1)}\right.$}

Instabilités provoquées par un système de régulation

Pompage d'un groupe à la suite de la rupture de l'asservissement aiguille-déflecteur (Usine de Sassenage)

A l'origine (en 1958) la conjugaison aiguilledéflecteur du groupe de Sassenage était réalisée par une transmission à huile qui avait l'inconvénient de se dérégler fréquemment en raison de la dilatation des tuyauteries.

Depuis 1968, un nouveau dispositif assure la conjugaison par l'intermédiaire d'un câble à billes qui transmet la position de l'aiguille au système d'asservissement grâce à un relais réducteur mécanique.

La connexion de ce relais, côté aiguille et côté déflecteur se fait par un ruban souple en acier de $15 \times 0,1 \mathrm{~mm}$.

Le déréglage (glissement sur les pièces de fixation) ou la rupture de l'un de ces rubans ou du câble à billes pro-

(1) Le texte intégral de cette communication et celui de la discussion peuvent être fournis par le Secrétariat de la S.H.F. 
voque la chute du déflecteur dans le jet et des mouvements intempestifs et rapides d'ouverture et de fermeture de l'aiguille qui peuvent créer des surpressions dangereuses dans la conduite forcée. Le phénomène est accompagné d'un bruit très intense (jet sur le déflecteur) qui est perçu même à l'extérieur de l'usine.

Entre le 30 janvier et le 22 février 1970, 7 incidents se sont produits, tous dus au glissement d'un ruban dans ses pièces de fixation.

Après l'incident du 22 février, une amélioration de la fixation des rubans semble avoir éliminé ce risque, mais on trouve ensuite, d'avril 1970 à janvier 1973, 4 incidents dus à la rupture d'un ruban.

Enfin, le 7 novembre 1976, s'est produit un nouvel incident qui a entraîné l'éclatement de la conduite forcée ; il a été attribué à la rupture du câble à billes de l'asservissement et du feuillard de ce câble :

La chronologie des faits a pu être reconstituée à l'aide, d'une part, des témoignages des personnes qui se trouvaient à proximité de la centrale au moment de l'incident, d'autre part, des enregistrements de la pression dans la conduite forcée et de l'E.M.S.

\section{Circonstances de l'incident}

Le niveau de la retenue atteignant la cote maximale, le groupe a reçu un ordre de démarrage. Après ouverture du robinet de garde, des oscillations se sont amorcées dans la conduite forcée pendant le couplage du groupe. L'examen des enregistrements de la pression dans la conduite forcée réalisés pendant un an, a montré que de telles oscillations se produisaient à chaque démarrage de l'usine et que leur amplitude était variable et pouvait atteindre \pm 8 bars, mais sur aucun des démarrages précédents, on n'avait constaté une amplification des oscillations par phénomène de résonance. Le 7 novembre, dès le début, l'amplitude a été importante et surtout elle a crû rapidement. Ce phénomène de résonance s'est poursuivi pendant 18 minutes environ et, au cours des dernières oscillations, la pression de 100 bars paraît avoir été dépassée. (Il n'est pas impossible qu'il y ait eu un lancer d'aiguille du manomètre).

Les témoins ont confirmé avoir entendu le bruit du pompage du groupe qui est allé en s'amplifiant et qui s'est terminé par la rupture de la conduite.

Après l'incident, la vitesse de déplacement du pointeau a été réduite afin que le phénomène de résonance ne puisse pas se reproduire; en outre un manostat a été installé sur la conduite forcée pour provoquer l'arrêt du groupe en cas de dépassement de la pression maximale admissible.

\section{Balancement d'une retenue par suite de la réflexion par la rive opposée des oscillations provoquées par la ma- noeuvre des clapets de réglage du niveau (Barrage des Fades)}

L'évacuateur de crues du barrage des Fades est équipé de 2 vannes-segments surmontées de clapets ; lorsque les débits à évacuer sont inférieurs à $96 \mathrm{~m}^{3} / \mathrm{s}$ le niveau du plan d'eau est réglé automatiquement par ces clapets qui agissent simultanément.
La mise au point de leur dispositif d'asservissement au niveau a été assez délicate par suite de la réflexion par la rive opposée des oscillations provoquées par les manœuvres.

Les clapets se manœuvrent actuellement par intermittence : déplacements de $0,25 \mathrm{~m}$ espacés de $35 \mathrm{mi}-$ nutes ; ce temps étant celui qui est mis par une oscillation pour faire un aller et retour, les ordres d'ouverture ou de fermeture sont donnés dans les mêmes conditions, ce qui assure un réglage stable. Par ailleurs, le puits du détecteur de niveau a été diaphragmé pour que les petites fluctuations du niveau ne perturbent pas les mesures.

\section{Instabilités provoquées par des tourbillons}

Vibrations d'un tronçon de conduite forcée compris entre deux joints de dilatation (Usine de Saint-Chamas)

Dès la mise en service de l'Usine de Saint-Chamas, on a constaté que, lorsque la puissance de l'une quelconque des 3 turbines était voisine de $50 \mathrm{MW}$, le double joint à ondes monté sur sa conduite forcée entre l'entonnement et la cheminée d'équilibre (voir Fig. 1) se mettait à vibrer à $4,8 \mathrm{~Hz}$ parallèlement à l'axe de la conduite. L'amplitude du mouvement de la virole comprise entre les 2 joints atteignait $48 \mathrm{~mm}$ entre crêtes. Un fonctionnement de quelques dizaines d'heures à ce régime a entraîné l'apparition de nombreuses fissures dans les soudures, nécessitant un arrêt pour réparation.

Des essais exécutés par la D.T.G. ont mis immédiatement hors de cause les turbines et montré que le joint oscillait sur sa période propre mécanique. Le système étant extrêmement peu amorti, il suffisait d'une force infime pour exciter les vibrations ; il est à peu près certain qu'elle était fournie par les tourbillons de l'écoulement au droit des ondes dont la surface intérieure n'est pas munie d'un guideau (voir Fig. 2).

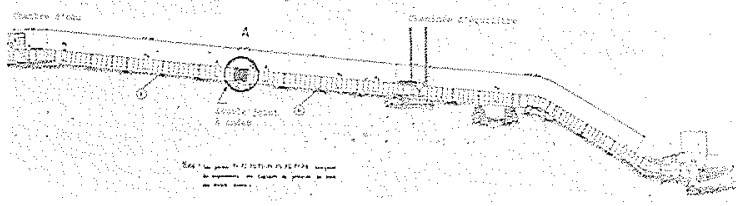

Figure 1 - St Chamas - Schéma de l'installation.

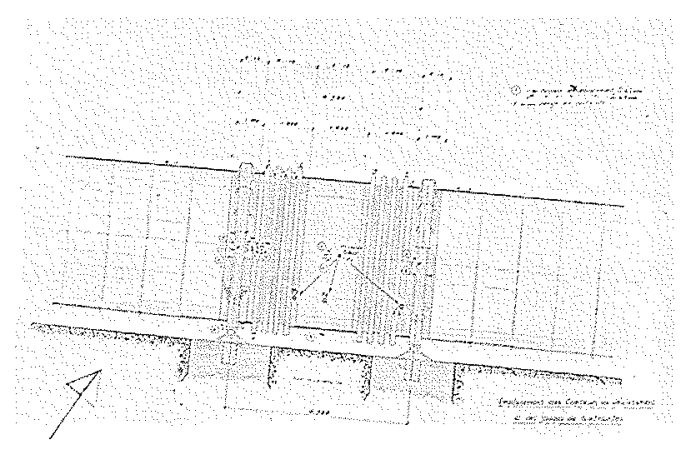

Figure 2 - St Chamas - Détail du double joint à ondes. 


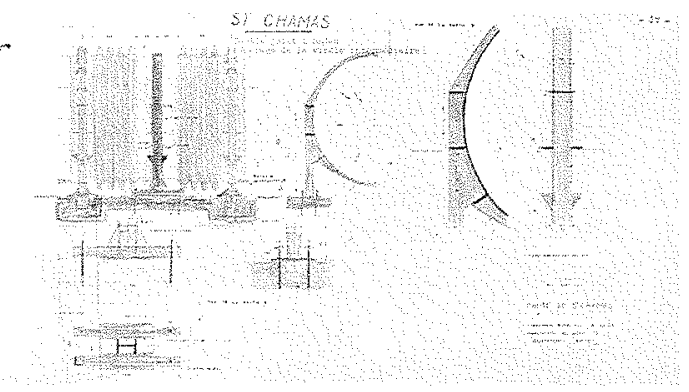

Figure 3 - St Chamas - Double joint à ondes. Ancrage de la virole intermédiaire.

Un lestage du joint n'a fait que déplacer la zone d'établissement des vibrations vers les faibles charges sans réduire les amplitudes car la fréquence des tourbillons est en première approximation proportionnelle à la vitesse de l'eau, donc grossièrement à la puissance.

Dans la solution définitive, essayée en septembre 1976 , la virole médiane a été liée au sol par un ancrage (voir Fig. 3). Aucune vibration n'est apparue depuis. Par ailleurs, il a été vérifié, au cours d'un remplissage de la conduite initialement chauffée par le soleil, que les différences de longueur et de régime thermique des deux tronçons de conduite encadrant le joint n'introduisaient aucun effort temporaire supérieur à celui calculé pour le régime définitif.

\section{Vibrations d'aubes avant-directrices (Usines de Ma- nosque et Sainte-Tulle II)}

Les Usines de Manosque et Sainte-Tulle II sont équipées chacune d'un groupe vertical ayant les caractéristiques suivantes:

- turbine Kaplan à 7 pales mobiles,

- puissance garantie sur l'arbre : $49000 \mathrm{~kW}$ sous $35,85 \mathrm{~m}$ de chute nette pour un débit de $151 \mathrm{~m}^{3} / \mathrm{s}$,

- vitesse de rotation : $150 \mathrm{tr} / \mathrm{mn}$,

- distributeur composé de 24 directrices mobiles en acier moulé A $56 \mathrm{M} 2$,

- avant-distributeur constitué de 2 anneaux en tôle en acier A 42.3 (épaisseur $140 \mathrm{~mm}$ ) reliés par un bec de volute et de 18 avant-directrices en tôle cintrée en acier A 42.3 soudées aux deux anneaux ; les bords d'attaque et de fuite des avant-directrices avaient à l'origine un profil demi-circulaire,

- dimensions des avant-directrices :

$\mathrm{n}^{\circ} 1$ à 17 : hauteur $1640 \mathrm{~mm}$, longueur $920 \mathrm{~mm}$, épaisseur $40 \mathrm{~mm}$.

$\mathrm{n}^{\circ} 18$ (près du bec de bâche) : hauteur $1650 \mathrm{~mm}$, longueur $480 \mathrm{~mm}$, épaisseur $40 \mathrm{~mm}$; rayon de courbure n ${ }^{\circ} 1$ à $18: 1.450 \mathrm{~mm}$.

Peu de temps après la mise en service des groupes, des bruits et des vibrations sont apparus au niveau des distributeurs : une visite détaillée des machines a montré l'existence de fissures de fatigue plus ou moins importantes au raccordement de certaines avant-directrices avec les plateaux des bâches.

Des essais ont alors été entrepris pour rechercher leur cause ; ils comportaient essentiellement des mesures

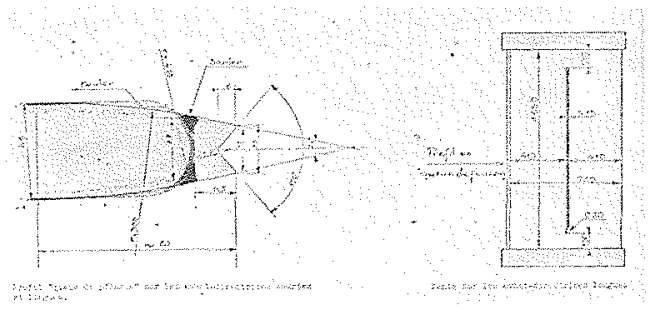

Figure 4 - Manosque et Ste Tulle Modifications des avant-directrices.

d'extensométrie en divers points de l'attache de quelques avant-directrices, complétées par des enregistrements de vibrations (axe des directrices) et de pressions (entrée, bâche, manteau en amont et en aval de la roue). Ils ont montré que le travail du métal en régime statique était normal et qu'en fonctionnement aucune instabilité hydraulique n'affectait l'ensemble des machines; la fissuration n'était imputable qu'à des vibrations des avant-directrices provoquées par deux phénomènes différents :

Le premier était la mise en résonance de l'avantdirectrice par les tourbillons de Karman se formant au niveau de l'arête de sortie ; de loin le plus dangereux, il intéressait toutes les avant-directrices (avant-directrice courte située près du bec de bâche et avant-directrices longues) ; il a été éliminé par une modification de l'arête de sortie: mise en place d'un profil dit "en queue de poisson" (voir Fig. 4).

Le second n'intéressait que les avant-directrices longues:

Alors que les avant-directrices courtes ne vibraient que suivant le mode de flexion à leur première fréquence propre (60 $\mathrm{Hz}$ dans l'eau), deux phénomènes étaient observés sur les avant-directrices longues :

- une vibration à $67 \mathrm{~Hz}$ analogue à la précédente résultant d'une excitation par tourbillons de Karman - une vibration à $95 \mathrm{~Hz}$ liée à l'écoulement autour du profil (oscillation de torsion)

L'adjonction d'un profil en "queue de poisson" sur l'arête de sortie, suffisante pour supprimer les vibrations des avants-directrices courtes, ne modifiait pas sensiblement les vibrations des avant-directrices longues. Ces dernières n'ont été supprimées que par la création d'une fente verticale au milieu de chaque avant-directrice (voir Fig. 4). Cette fente a eu pour effet de désolidariser l'entrée et la sortie du profil (d'où meilleur amortissement des perturbations de l'écoulement dues aux mouvements de l'avant-directrice) et surtout de diminuer la fréquence propre dans le mode de torsion.

En résumé, les solutions retenues, ont été : “queue de poisson" sur toutes les avant-directrices et fente sur les avant-directrices longues.

Elles ont été identiques pour les deux usines bien que les deux groupes n'étant pas de construction rigoureusement identique, les observations faites sur chacun d'eux n'aient pas été tout à fait les mêmes.

Actuellement, il ne subsiste que quelques vibrations de l'avant-directrice courte de Manosque entre 25 et $30 \mathrm{MW}$, apparement sans danger mais que l'on évite de provoquer en l'absence de sujétions d'exploitation. 


\section{Instabilités provoquées par des vannes sur conduites}

Oscillations de pression dans des conduites provoquées par les vannes sphériques d'isolement d'un collecteur (Usine de Pragnères)

La figure 5 représente le schéma hydraulique général de l'aménagement et la position des deux vannes $A$ et $B$ du collecteur. Une anomalie de conception entachait le système d'étanchéité de celles-ci : l'anneau mobile qui devait assurer l'étanchéité en se serrant sur l'opercule se trouvait en équilibre indifférent lorsque les pressions amont et aval étaient équilibrées ce qui était le cas lors d'une manœuvre. L'ordre de fermeture ne modifiait en rien cet équilibre indifférent et on constatait que, pour obtenir le déplacement de l'anneau mobile et l'étanchéité de la vanne, il fallait créer une dépression importante à l'aval de celle-ci.

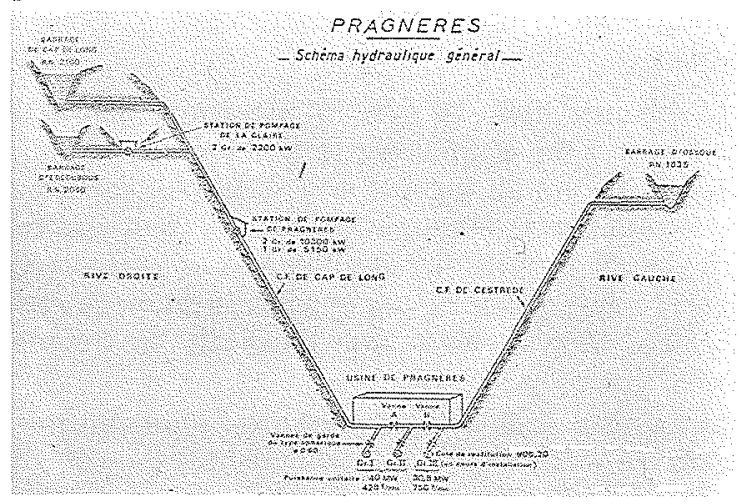

Figure 5 - Pragnères - Schéma hydraulique général.

Cette anomalie s'est révélée extrêmement dangereuse pour l'installation car un phénomène d'oscillation prenait naissance dans les conduites rive droite et rive gauche à l'occasion des mancuvres de sectionnement du collecteur ; au cours de certaines d'entre elles la pression variait de 720 à $1150 \mathrm{~m}$ pour une pression de service de $930 \mathrm{~m}$.

Le constructeur a été conduit à remplacer les anneaux d'étanchéité en équilibre par des anneaux différentiels qui donnent maintenant entière satisfaction.

\section{Instabilités provoquées par des turbines}

Vibrations d'une conduite forcée au cours des démarrages en pompe et en turbine (Usine de La Coche)

\section{Généralités}

L'aménagement de La Coche mis en service en 1977 est un aménagement mixte pompage-gravitaire. Il comporte une conduite forcée dont la longueur totale est de $2000 \mathrm{~m}$ et le diamètre intérieur variable de $3,20 \mathrm{~m}$ à $2,55 \mathrm{~m}$. Cette conduite alimente 4 groupes réversibles tournant à $600 \mathrm{tr} / \mathrm{mn}$ et comportant chacun :

- 1 pompe-turbine sans organe de réglage capable de turbiner $9,6 \mathrm{~m}^{3} / \mathrm{s}$ sous $930 \mathrm{~m}(P=79,5 \mathrm{MW})$ et de pomper $8,7 \mathrm{~m}^{3} / \mathrm{s}$ sous $882 \mathrm{~m}(P=83,0 \mathrm{MW})$

- 1 alternateur-moteur de $85 \mathrm{MVA}-15,5 \mathrm{kV}$

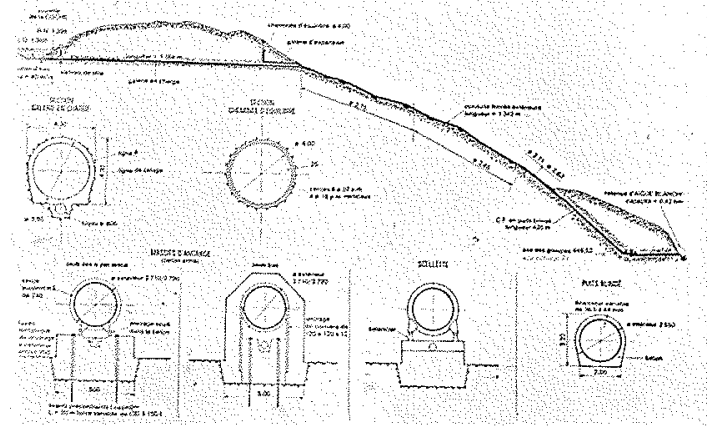

Figure 6 - La Coche - Schéma de l'installation.

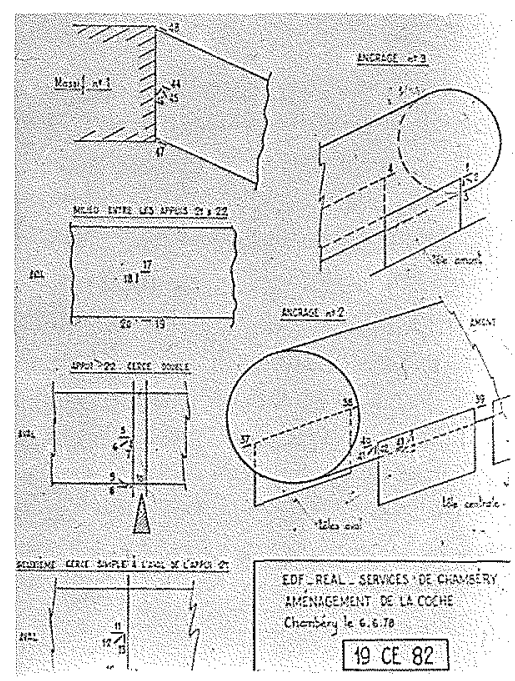

Figure 7 - La Coche - Conduite forcée. Implantation des jauges.

La réalisation de la conduite forcée dans sa partie extérieure longue de $1370 \mathrm{~m}$, a été facilitée par l'adoption d'un montage après exécution complète du génie civil. C'est ainsi que dans les massifs ont été incluses 2 plaques verticales sur lesquelles la conduite a été fixée (voir Fig. 6 et 7).

Pendant la phase de démarrage en turbine et en pompe, il a été constaté que la conduite vibrait. La Région d'Equipement a alors cherché :

- à évaluer les contraintes dans les tôles de la conduite et des plaques d'ancrage de celle-ci dans les massifs,

- le moyen de réduire les contraintes dues aux vibrations à un niveau acceptable.

\section{Evaluation des contraintes}

A la demande de la Région, la D.T.G. a procédé à des mesures de contraintes par jauges extensomètriques collées en différents points caractéristiques: contre les massifs en béton, contre les plaques d'ancrage, près des cerces doubles ou simples et en deux points courants.

Ces mesures ont été faites pour les régimes suivants:

- démarrage en turbine,

- fonctionnement avec obturateur fermé,

- ouverture de l'obturateur et montée en puissance,

- marche à la puissance nominale et arrêt,

- démarrage en pompe,

- refoulement avec anneau ouvert et obturateur fermé, 
- refoulement avec obturateur ouvert,

- marche à la puissance nominale et arrêt.

Ces essais ont été réalisés avec le groupe I et le groupe III fonctionnant simultanément ou séparément.

Les contraintes vibratoires, engendrées par les manœuvres du groupe III, sont de même nature mais sensiblement moins importantes que celles provoquées par le groupe I à même condition de marche. Pour le même point d'ancrage on relève 3,5 à 5 hbar pour le G III contre 4 à 7,3 hbar pour le $G$ I en démarrage normal et 3,5 à 8,1 hbar (G III) contre 5,4 à 14,6 hbar (G I) en marche sur anneau ouvert et obturateur fermé.

Les contraintes maximales relevées sur l'un ou l'autre groupe en démarrage normal sont sensiblement moins importantes que celles relevées en marche sur obturateur fermé. De ce fait, les valeurs extrêmes données cidessus doivent être considérées comme des maxima qui ne seraient atteints qu'en cas de non ouverture du sphérique.

Le démarrage de l'un ou de l'autre des groupes, l'autre étant en service, n'apporte pas de modification sensible, ce régime correspondant au démarrage du groupe seul.

Les valeurs maximales des contraintes vibratoires dans la tôle de la conduite sont à une fréquence voisine de $10 \mathrm{~Hz}$.

Dès que l'ouverture de l'obturateur dépasse environ $25 \%$ les fluctuations diminuent rapidement pour atteindre des niveaux inférieurs ou égaux à 1 hbar crête à crête sur toutes les jauges.

Ces enregistrements font apparaître des phénomènes de battements sur les jauges les plus sollicitées. La période de ces battements varie suivant les jauges.

En conclusion, les essais ont montré que :

- des contraintes vibratoires se développent dans des zones très localisées n'intéressant que les parties où la conduite rencontre la première tôle d'appui. Elles atteignent des valeurs maximales crête à crête de 14,6 hbar (marche avec obturateur fermé),

- au voisinage d'un ancrage réalisé par un massif de béton entournant toute la conduite, les contraintes sont tout à fait acceptables ( 1 à 2 hbar crête à crête)
- en pleine tôle ou au voisinage des cerces, les contraintes sont également acceptables. L'effet de rigidification des cerces doubles et des appuis est assez marqué puisque les contraintes sont de l'ordre de 4 hbar.

\section{Diminution des contraintes}

Pour ramener les contraintes vibratoires dans la tôle de la conduite à un niveau acceptable, deux expériences de modifications des ancrages des conduites sur ses appuis ont été tentées:

- mise en place d'une cerce simple de renfort à l'aval du massif $n^{\circ} 2$.

- élargissement des tôles support et goussets à l'amont du massif $n^{\circ} 3$.

Les mesures faites au cours de démarrages en pompe avec ouverture du seul anneau d'étanchéité, avec cerce serrée non soudée, cerce serrée et soudée sur plaque d'ancrage et cerce soudée sur la moitié de la conduite et sur plaques d'ancrage ont permis d'approcher la solution définitive : cerce soudée et élargissement des supports.

Toutefois, comme la cerce mise en place laissait subsister des contraintes importantes dues à son inertie relativement faible, il a été décidé de mettre en place une cerce renforcée. L'usine étant arrêtée pendant une longue période, et afin d'avoir un ordre de grandeur de l'efficacité de cette dernière cerce, il a été procédé à l'excitation de la conduite vide et à une mesure comparative des contraintes mesurées avec la première cerce et avec la cerce renforcée.

Ces essais ont permis de constater :

- l'amélioration de l'encastrement grâce à la cerce renforcée (en $\mathrm{H}$ )

- la division par 2 de la contrainte aux extrêmités des goussets.

Il a donc été décidé d'adopter ces cerces renforcées.

Les mesures effectuées en Mai 1978 par la D.T.G. ont permis de constater une nette amélioration : la contrainte maximale est passée de 12 à 2,5 hbar.

\section{Discussion}

Président : M.J. CHEVALIER

M. CHEVALIER. - Merci M. PLICHON. Cet exposé nous laisse sur la même faim que vous. Effectivement, quand de telles mésaventures se produisent, l'expérience montre qu'on s'en tire par un empirisme raisonné. Il n'en reste pas moins que c'est un coup au coeur pour le mécanicien quand il s'aperçoit que la solution est venue en ajoutant des ancrages, mais sans s'expliquer pourquoi il y a vibration et sans savoir ce qu'il faudrait faire pour que le problème ne se pose pas.

M. CHAUBERT. - J'aimerais non pas donner une explication, mais citer un cas intéressant de mise en vibration par tourbillons de Karman qui, peut-être, complètera l'exposé de $M$. PLICHON.

Nous avons fourni pour l'Aménagement du Sarganserland, en Suisse orientale, 3 vannes papillons avec obturateur dit "en treillis", d'un diamètre nominal de $3,50 \mathrm{~m}$

L'aménagement comprend 3 groupes ternaires de $93 \mathrm{MW}$, dont les turbines débitent ensemble $75 \mathrm{~m}^{3} / \mathrm{s}$ et les pompes $36 \mathrm{~m}^{3} / \mathrm{s}$.

Deux vannes papillons, en série, sont situées à proximité de la prise d'eau du bassin supérieur. La $3^{\mathrm{e}}$ est placée en tête du puits blindé, séparée des deux autres par $7 \mathrm{~km}$. de galerie.

Les 3 vannes, à part les organes de commande, sont de construction identique. L'obturateur est constitué par une plaque circulaire, épaisse de $80 \mathrm{~mm}$, portant sur son pourtour un joint d'étanchéité en néoprène. Cette plaque est soudée sur des têtes en acier moulé, sur lesquelles les tourillons sont vissés. Une seconde plaque, rectangulaire, assure la rigidité. Elle est liée à la première par des entretoises en tôle relativement minces (30 et $40 \mathrm{~mm}$ ).

Dès la mise en service de la $2^{e}$ turbine, une vibration s'est manifestée, de manière identique sur les 3 vannes, par un bourdonnement assez fort de fréquence $52 \mathrm{~Hz}$. Cela commençait 
vers $40 \mathrm{~m}^{3} / \mathrm{s}$, l'amplitude maximum se situait vers $45 \mathrm{~m}^{3} / \mathrm{s}$, puis brusquement le phénomène disparaissait et à $52 \mathrm{~m}^{3} / \mathrm{s}$, il n'y avait plus rien.

Lorsque, quelques mois plus tard, les 3 turbines furent mises en service, une nouvelle vibration apparut dès $62 \mathrm{~m} 3 / \mathrm{s}$, mais à la fréquence de $82 \mathrm{~Hz}$, le maximum d'amplitude se trouvant aux environs de $70 \mathrm{~m}^{3} / \mathrm{s}$.

Les parties extérieures des corps de vanne ne vibraient que très faiblement, et le phénomène était, somme toute, plus curieux que vraiment alarmant.

Nous avons immédiatement pensé aux effets des tourbillons de Karman, provoquant la mise en résonance de certaines parties de l'obturateur. Il est connu que la fréquence des tourbillons est proportionnelle à la vitesse de l'eau. Or, les deux fréquences en question étaient bien dans le même rapport que les vitesses.

Le calcul des fréquences propres de vibration d'une pièce telle que notre obturateur, même en utilisant des méthodes très évoluées, ne pouvait guère donner que des résultats assez vagues, du fait des hypothèses assez fragiles, quant aux liaisons entre les différents éléments de la construction. De même, le calcul des tourbillons de Karman, basé sur un nombre de Strouhal difficile à chiffrer, ne donne qu'un ordre de grandeur de leur fréquence.

Pour analyser correctement le phénomène, il était nécessaire de faire des mesures à l'intérieur de l'une des vannes, pendant la marche, et sous tous les débits possibles. Pour cela, 24 jauges à résistance ont été collées en divers points de l'obturateur, bien entendu soigneusement isolées par une résine étanche et reliées aux appareils extérieurs par une torche de câbles souples, permettant de manoeuvrer l'obturateur, et assez solides pour résister au courant d'eau.

La vibration enregistrée est une sinusoüde presque dépourvue d'harmoniques. Comme prévu, les contraintes alternées ainsi mesurées étaient faibles, et la fatigue des éléments vibrants, les entretoises, n'était pas à craindre. Nous avons toutefois jugé nécessaire de tout tenter pour faire disparaître ce phénomène.

Les bords de fuite des tôles étaient profilés, suivant une forme qui nous avait paru correcte avec un arrondi de 5 à $6 \mathrm{~mm}$ de rayon. En vue de diminuer l'amplitude et de changer la fréquence des tourbillons, nous avons soudé, simplement sur ces bords de fuite, des barres de section beaucoup plus pointue.

Cette petite modification, qui a été effectuée jusqu'à maintenant à titre d'essai sur l'une des vannes, a donné un résultat absolument concluant. Quel que soit le débit, la vanne est maintenant silencieuse. Les deux autres vannes vont être modifiées de la même manière dès que les conditions d'exploitation le permettront.

M. CHEVALIER, - Merci Monsieur, voilà un exemple qui rejoint tout à fait ce qu'a fait M. PLICHON.

M. LACOSTE. - En complément des cas signalés par M. PLICHON, je voudrais souligner les points suivants :

Les phénomènes de résonance hydraulique sur la fréquence propre de la conduite forcée sont relativement fréquents et toujours dangereux : phénomènes de clapets battant düs à des mouvements de joints mobiles de vanne sphérique, par exemple, ou comme cela s'est produit lors du démarrage d'un groupe Pelton d'ORLU, oscillation de l'ensemble régulateur-pointeau, la turbine étant en marche à vide, sur la fréquence propre de la conduite forcée. Ces phénomènes sont d'autant plus dangereux que les débits étant faibles, l'amortissement par pertes de charge est négligeable et le "coefficient de surtension" à la résonance élevé.

En ce qui concerne les vibrations engendrées par les turbines, je voudrais signaler un cas curieux. Il s'agit du problème posé à l'ARGENTIERE par une turbine équipée d'une roue Francis double dont les aubages de chaque partie de la roue étaient en face les uns des autres au lieu d'être quinconcés comme ils le sont habituellement. La turbine équipée de cette roue provoquait des vibrations importantes à $150 \mathrm{~Hz}$, qui ébranlaient la conduite forcée et même le génie civil de la centrale. Ces vibrations étaient causées par les ébranlements dûs au passage des pales devant les directrices qui, par suite de leur décalage dans le temps et dans l'espace et de la forme géométrique de la bâche se "focalisaient" à l'entrée de celle-ci. Le remplacement de la roue par une autre de même profil d'aubage, mais dont les arêtes d'entree étaient en quinconce a pratiquement supprimé les vibrations à $150 \mathrm{~Hz}$.

Tous les phénomènes signalés jusqu'ici par M. PLICHON et moi-même sont périodiques. Pour terminer, je voudrais rappeler un phénomène non périodique que j'ai déjà évoqué lors d'une séance précédente de la S.H.F. Il s'agit de modifications réversibles constatées sur les caractéristiques de rendement et vraisemblablement de débit de certains Bulbes. La Production Hydraulique, et plus précisément la D.T.G., étudie actuellement ce curieux phénomène. Nous avons la conviction qu'il ne s'agit pas de perturbations dues à des corps étrangers, mais qu'il s'agit de 2 régimes hydrauliques de fonctionnement et nous poursuivons les investigations nécessaires pour en découvrir les causes.

M. CHEVALIER, - Merci, M.LACOSTE.

M. CRAVERO. - Voici quelques informations complémentaires concernant les problèmes rencontrés à St-CHAMAS.

A St-CHAMAS, les essais ont été conduits par étapes en éliminant successivement la turbine comme excitateur possible (rien ne distingue à son niveau les fortes charges, où se produit le phénomène, de la charge optimale) puis l'ensemble des ouvrages d'amenée. Une étude de l'évolution des pressions le long des conduites ne mettait en évidence aucun phénomène se propageant à la fréquence rencontrée.

Restait donc la seule possibilité d'une localisation au joint à onde. M. PLICHON a dit comment une modification de masse avait à la fois diminué la fréquence propre et fait se développer le phénomène à sa nouvelle fréquence pour une puissance, donc une vitesse d'écoulement, inférieure. L'hypothèse d'une relation avec les tourbillons de Karman était donc fort tentante. Par l'étude du facteur d'amortissement du système qui conduisait à un coefficient de résonnance de 400 , on montrait que leur énergie était suffisante pour pouvoir exciter le système.

La mise en place d'un guide-eau, comme c'est souvent le cas dans de tels joints, eut apporté la réponse définitive, mais une solution industrielle plus simple ayant été trouvée, l'expérience n'a pas été tentée.

M. CANA VELIS. - Dans l'exemple de la mise en résonance de la manchette de SAINT-CHAMAS, on peut se demander si les phénomènes observés ne sont pas liés au changement brusque de la vitesse de propagation des ondes à travers la manchette qui présente une élasticité différente de celle des conduites métalliques.

Dans ces conditions, les variations de pression qui apparaissent dans la conduite subissent un déphasage aux bornes de la manchette et peuvent, de ce fait, engendrer des efforts longitudinaux variables sur cet élément.

M. PLICHON. - En "ficelant" sur la manchette on a changé la masse, mais pas la rigidité

Je vous signale d'ailleurs que la vitesse de propagation dans la conduite, qui avait un gros diamètre, était très inférieure à $1.000 \mathrm{~m} / \mathrm{s}$ et plus proche de $500 \mathrm{~m} / \mathrm{s}$.

M. ROUQUIE. - Nous avons eu l'occasion de faire des études assez approfondies sur le comportement des compensateurs de dilatation à ondes. On s'est aperçu que pour certaines vitesses d'écoulement, il peut se créer des tourbillons qui ont une longueur de corrélation importante : on a pratiquement un tourbillon captif et il peut être synchronisé d'une onde à l'autre à certaines vitesses d'écoulement, si bien qu'avec un écoulement à faible énergie, on arrive à provoquer des déplacements, et les déplacements réagissent sur la formation des tourbillons. Le tout se synchronise.

Et l'on peut, comme vous l'avez fait, maîtriser ce phénomène. Mais on peut également le contrôler en rapportant un produit amortissant sur les ondes elles-mêmes : c'est ce que nous avons fait sur des installations très importantes.

M. CHEVALIER. - Vous avez dit en appliquant un produit amortissant ?

M. ROUQUIE. - Oui, il suffit de caoutchouc par exemple qui amortit par frottement.

Ceci suppose une résonance mécanique importante, ce qui fait qu'un très faible amortissement suffit, mais il y a aussi l'interaction entre la formation du tourbillon et le déplacement des ondes.

M. CHEVALIER. - Je remercie M. PLICHON. 